\title{
microRNA-124-3p inhibits the progression of congenital hypothyroidism via targeting programmed cell death protein 6
}

\author{
WENJIE LI, DONGPO SONG, YINGMEI SUN, YANAN LV and JINFENG LV \\ Newborn Screening Center, Qingdao Women and Children's Hospital, Qingdao, Shandong 266034, P.R. China
}

Received August 30, 2017; Accepted December 8, 2017

DOI: $10.3892 /$ etm.2018.6062

\begin{abstract}
The incidence of congenital hypothyroidism $(\mathrm{CH})$ in newborn infants ranges from 1 in 3,000 to 1 in 4,000. Previous studies have indicated the neuroprotective role of microRNA (miR)-124-3p, however the expression and role of miR-124-3p in $\mathrm{CH}$ remain unclear. Therefore, the present study was performed to investigate the role and precise molecular mechanism of miR-124-3p in $\mathrm{CH}$. Propylthiouracil (50 mg/day) was injected into the stomach of pregnant rats from gestational day 15 until parturition in order to establish a thyroid hypofunction model. Newborn rats were divided into the following four groups: The control group; the thyroid hypofunction group; the miR-124-3p mimic group; and the miR-124-3p negative control group. Reverse transcription-quantitative polymerase chain reaction indicated that miR-124-3p was significantly decreased in the hippocampus of the thyroid hypofunction group compared with the control group. Bioinformatics software was used to predict mRNA targets recognized by miR-124-3p and the programmed cell death protein 6 (PDCD6) 3' untranslated region (UTR) was demonstrated to exhibit the seed sequence of miR-124-3p. The interaction between miRNA-124-3p and PDCD6 was then verified using a dual-luciferase reporter assay system. PDCD6 expression was significantly increased in the hippocampus of rats with $\mathrm{CH}$ compared with the control group. Flow cytometry was performed to investigate the effects of miR-124-3p on neuronal cell apoptosis and the results indicated that the apoptosis rate in the thyroid hypofunction group was significantly increased compared with the control group; this increase was reversed by transfection with miR-124-3p mimics. Western blot analysis was used to detect the levels of cleaved poly [ADP-ribose] polymerase (PARP), full-length PARP, caspase-3, B cell lymphoma-2 (Bcl-2) and $\mathrm{Bcl}-2$-associated $\mathrm{X}$ protein (Bax) proteins. The results
\end{abstract}

Correspondence to: Dr Wenjie Li, Newborn Screening Center, Qingdao Women and Children's Hospital, 6 Tongfu Road, Qingdao, Shandong 266034, P.R. China

E-mail: liwenjie171230@163.com

Key words: congenital hypothyroidism, microRNA-124-3p, programmed cell death protein 6 , apoptosis indicated that the expression of cleaved PARP, caspase-3 and Bax protein were significantly increased and the expression of full-length PARP and Bcl-2 protein was significantly decreased compared with the control group. These effects were reversed by miRNA-124-3p mimic transfection. Taken together, the results of the present study demonstrate that miRNA-124-3p serves a protective role in $\mathrm{CH}$ via targeting PDCD6.

\section{Introduction}

Congenital hypothyroidism $(\mathrm{CH})$ is defined as thyroid hormone deficiency at birth and is one of the most frequent preventable causes of mental retardation; however, the majority of infants with $\mathrm{CH}$ do not exhibit obvious clinical manifestations at birth (1). In hypothyroidism, type 2 deiodinase is adapted to increase the conversion of the prohormone thyroxine to the biologically active triiodothyronine (2). Thyroid hormone serves a pivotal role in the development of the mammalian brain and its dyssynthesis, paracrisis or lack of biological effects may be caused by hypothyroidism (3). $\mathrm{CH}$ results in various developmental disorders (4). The incidence in newborn infants ranges from 1 in 3,000 to 1 in 4,000 (5) and is markedly higher in preterm compared with full term infants (6).

Previous studies have demonstrated the importance of timely treatment for neurologic outcome, which was indicated by an inverse correlation between intelligence quotient and the age of diagnosis $(7,8)$. Despite early diagnosis of $\mathrm{CH}$, neurologic maldevelopment may also occur when treatment is not optimized in the first 2-3 years of life (9). It is therefore important for patients with $\mathrm{CH}$ to receive early treatment and close follow up.

MicroRNAs (miRNAs or miRs) are short non-coding RNAs, which regulate post-transcriptional gene silencing (10). Thousands of miRNAs have been identified in a variety of species (11). Binding of miRNA to the $3^{\prime}$ untranslated region (UTR) may degrade target mRNA and block translation (12). Previous studies have suggested the role of miRNAs in nervous system diseases, including autoimmune neuroinflammation (13), Alzheimer's disease (14) and Parkinson's disease (15). miR-124-3p may also attenuate matrix metalloproteinase-induced neuronal injury via regulating signal transducer and activator of transcription 3 expression in SH-SY5Y cells (16). Huang et al (17) suggested 
that miR-124-3p may inhibit neuronal inflammation induced by traumatic brain injury and contributes to neurite outgrowth. Furthermore, miR-124-3p serves a neuroprotective role in the 6-hydroxydopamine-induced cell model of Parkinson's disease via regulating ANAX5 (18). miR-124 was also demonstrated to protect neurons from apoptosis in newborn rats with thyroid hypofunction (19). However, the expression and role of miR-124-3p in thyroid hypofunction remains unclear. Therefore, the present study was performed to investigate the role and precise molecular mechanism of miR-124-3p in $\mathrm{CH}$.

\section{Materials and methods}

Animal model. A total of 12 pregnant Sprague Dawley rats (body weight, $200 \pm 10 \mathrm{~g} ; \sim 6$ week old) were kept in Qingdao Women and Children's Hospital (Shandong, China) in appropriate-sized cages at room temperature and a humidity of $55 \%$. Mice were subjected to a $12 \mathrm{~h}$ light/dark cycle and had ad libitum access to standard pellet feed and water. Propylthiouracil $(50 \mathrm{mg} /$ day; Beyotime Institute of Biotechnology, Haimen, China) was injected intraperitoneally into pregnant rats at gestational day 15 each day until parturition in order to establish the thyroid hypofunction rat model as previously described (20). Following anesthetization with intraperitoneal injection of $2 \%$ pentobarbital sodium (40 mg/kg), newborn rats (12 days old) were fixed on stereotaxic apparatus and their skulls were opened at $1.0 \mathrm{~mm}$ from the former fontanel and $1.7 \mathrm{~mm}$ from the mid-line. Thereafter, a micro syringe was inserted vertically $3.8 \mathrm{~mm}$ at $15 \mu \mathrm{m} / \mathrm{sec}$ and mice were injected with $5 \mu \mathrm{l}$ $1 \mathrm{nmol} / 1 \mathrm{miR}-124-3 \mathrm{p}$ mimic solution or miR-124-3p negative control (NC) solution (Shanghai GenePharma Co., Ltd., Shanghai, China) as previously described (21). Newborn rats were divided into the following four groups (each $n=5)$ : The control group, the thyroid hypofunction group, the miR-124-3p mimic group and the miR-124-3p NC group. The present study was approved by the Animal Ethics Committee of Qingdao Women and Children's Hospital (Qingdao, China).

Reverse transcription-quantitative polymerase chain reaction $(R T-q P C R)$. At day 15 following birth, newborn rats were sacrificed and the hippocampus was harvested. Total RNA was extracted from the hippocampus using a PrimeScript reverse transcription reagent kit (Takara Biotechnology Co., Ltd., Dalian, China) in accordance with the manufacturers' protocols. cDNA was synthesized by RT using the following primers: miR-124-3p, 5'-GTCGTATCCAGTGCAGGGTCC GAGGTATTCGCACTGGATACGACGGCATT-3'; and U6, 5'-AAAATATGGAACGCTTCACGAATTTG-3'. PCR was conducted using the following primers: miR-124-3p, forward, 5'-GCTAAGGCACGCGGTG-3' and reverse, 5'-GTGCAG GGTCCGAGGT-3'; U6, forward 5'-CTCGCTTCGGCA GCACATATACT-3' and reverse 5'-ACGCTTCACGAATTT GCGTGTC-3'; PDCD6, forward 5'-ATGGCCGCCTACTCT TACC3' and reverse 5'TCCTGTCTTTATCGACCCTCT G3'; GAPDH, forward 5'CTTTGGTATCGTGGAAGGACT C3' and reverse 5'GTAGAGGCAGGGATGATGTTCT3'. The thermocycling conditions were as follows: $5 \mathrm{~min}$ at $95^{\circ} \mathrm{C}$ and
40 cycles of $30 \mathrm{sec}$ at $95^{\circ} \mathrm{C}, 30 \mathrm{sec}$ at $60^{\circ} \mathrm{C}$ and $30 \mathrm{sec}$ at $72^{\circ} \mathrm{C}$. Gene expression was normalized to U6 with using the $2^{-\Delta \Delta \mathrm{Cq}}$ method (22). This experiment was performed in accordance with a previous study (19).

Cell culture. Neurons were isolated from the hippocampus of rats in the four groups. The cerebral cortices of rats were dissected under an inverted microscope (Olympus Corporation, Tokyo, Japan; magnification: x40). Neurons were seeded in poly-D lysine-coated plates at a concentration of $1 \times 10^{6}$ cells $/ \mathrm{ml}$ and cultured in Dulbecco's modified Eagle's medium (Gibco; Thermo Fisher Scientific, Inc., Waltham, MA, USA) and 10\% fetal bovine serum (Gibco; Thermo Fisher Scientific, Inc.) for $4-6 \mathrm{~h}$ at $37^{\circ} \mathrm{C}$. Subsequently, the cell culture medium was replenished with neurobasal medium (Gibco; Thermo Fisher Scientific, Inc.), 2\% B27, $100 \mathrm{U} / \mathrm{ml}$ penicillin, $100 \mathrm{~g} / \mathrm{ml}$ streptomycin and $0.5 \mathrm{mM}$ glutamine (Gibco; Thermo Fisher Scientific, Inc.) and neurons were cultured in a incubator at $37^{\circ} \mathrm{C}$ in an atmosphere containing $5 \% \mathrm{CO}_{2}$. The culture medium was changed every 3 days.

Transfection with miRNA mimics. Neurons were seeded in 6-well plates $\left(5 \times 10^{4}\right.$ cells per well) and transfected with $50 \mathrm{nM}$ miR-124-3p mimics or $50 \mathrm{nM}$ miR-124-3p negative control (NC; both Shanghai GenePharma Co., Ltd.) using Lipofectamine ${ }^{\circledR} 2000$ (Thermo Fisher Scientific, Inc.) according to the manufacturer's protocol, following the attainment of 60-70\% confluence. After $24 \mathrm{~h}$, neurons were collected for subsequent experiments.

Flow cytometry. Cell apoptosis and the cell cycle were analyzed in the present study using a flow cytometer (FCM; BD Biosciences, Franklin Lakes, NJ, USA). Neurons $\left(1 \times 10^{5}\right.$ cells/well) were digested with $0.025 \%$ trypsin, washed with PBS and fixed in $70 \%$ cold ethanol at $4^{\circ} \mathrm{C}$ overnight. Subsequently, propidium iodide (PI, Sigma Aldrich; Merck KGaA, Darmstadt, Germany), RNaseA and 0.2\% Triton X-100 were added to cells and incubated at $4^{\circ} \mathrm{C}$ for $30 \mathrm{~min}$ in the dark.

Neurons were adjusted to give a concentration of $1 \times 10^{6}$ cells $/ \mathrm{ml}$ prior to the flow cytometry assay. The cell apoptosis rate was detected at $1 \mathrm{~h}$ following the addition of $5 \mu \mathrm{l}$ fluorescein isothiocyanate-labeled Annexin V and $5 \mu \mathrm{l}$ PI (cat. no. 6592; Cell Signaling Technology, Inc., Danvers, MA, USA). The cell number at each phase was analyzed using FlowJo software 7.6.1 (Tree Star Inc., Ashland, OR, USA).

Western blot analysis. At $24 \mathrm{~h}$ post-transfection, neurons were digested with lysis buffer (Cell Signaling Technology, Inc.). Proteins were quantified using a BCA assay (Thermo Fisher Scientific, Inc.). Total protein (25 $\mu \mathrm{g}$ per lane) was separated by $10 \%$ SDS-PAGE, transferred to polyvinylidene fluoride membranes and blocked with $5 \%$ bovine serum at room temperature for $1.5 \mathrm{~h}$. Membranes were incubated with primary antibodies against caspase-3 (cat. no. 9665), B-cell lymphoma 2 (Bcl-2; cat no. 4223), Bcl-2-associated X protein (Bax; cat. no. 5023), cleaved poly ADP-ribose polymerase (PARP; cat. no. 5625) and PARP (cat. no. 9532) (all 1:1,000; all Cell Signaling Technology, Inc.) at $4^{\circ} \mathrm{C}$ overnight. $\beta$-actin (cat. 


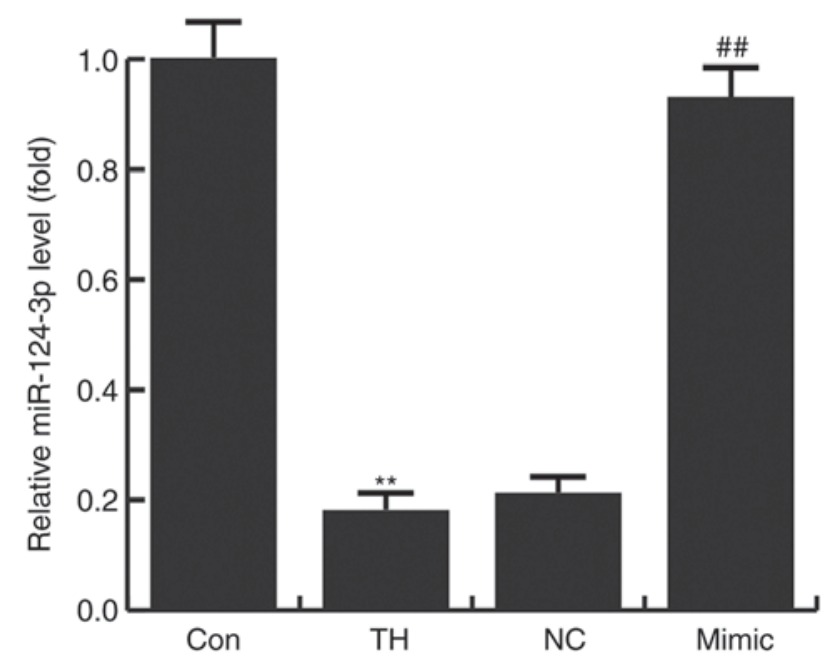

Figure 1. miR-124-3p was downregulated in rats with $\mathrm{CH}$. Values are expressed as the mean \pm standard deviation. Tests were performed in triplicate. ${ }^{* *} \mathrm{P}<0.01$ vs. the control group; ${ }^{\# \#} \mathrm{P}<0.01$ vs. the TH group. miR-124-3p, microRNA-124-3p; CH, congenital hypothyroidism; con, the control group; TH, the thyroid hypofunction group; NC, the miR-124-3p NC group; mimic, the miR-124-3p mimics group.

no. 4970; 1:5,000; Cell Signaling Technology, Inc.) was used as an internal control. Membranes were then incubated with horseradish peroxidase-conjugated secondary antibodies (cat. no. ab191866 and ab218695; 1:1,000; Abcam, Cambridge, UK) at room temperature for $1 \mathrm{~h}$. Bands were quantified using enhanced chemiluminescence (cat. no. 6883; Cell Signaling Technology, Inc.). Each protein level was normalized to $\beta$-actin (cat. no. 4970). The band density was quantified with Gel-Pro Analyzer densitometry software (Version 6.3, Media Cybernetics, Inc., Rockville, MD, USA).

Dual luciferase reporter assays. Bioinformatics software (http://www.microrna.org/microrna/home.do and http://www. targetscan.org/vert_71/) was used to predict the potential targets of miR-124-3p and programmed cell death protein 6 (PDCD6) was identified as a promising target of miR-124-3p. The vectors named PDCD6-3'UTR-wild type (WT) and PDCD6-3'UTR-mutant (MUT) (GeneCopoeia, Inc., Rockville, MD, USA) with wild type and mutated 3'UTRs of PDCD6 mRNA were used to investigate whether miR-124-3p directly targets the 3 'UTR of PDCD6. Cells $\left(5 \times 10^{4}\right.$ cells per well) were seeded in 24-well plates and co-transfected with PDCD6-3'UTR-WT (2 $\mu \mathrm{g})$ or PDCD6-3'UTR-MUT $(2 \mu \mathrm{g})$ plasmid and miR-124-3p mimics (50 $\mathrm{nM}$ ) or miR-124-3p NC (50 nM) using Lipofectamine ${ }^{\circledR} 2000$ (Thermo Fisher Scientific, Inc.). After $24 \mathrm{~h}$, relative firefly and Renilla luciferase activities were detected using a dual luciferase reporter assay kit (Promega Corporation, Madison, WI, USA) in accordance with the manufacturer's protocol. Firefly luciferase activity was used as a control.

Statistical analysis. Statistical analyses were conducted using SPSS 16.0 (SPSS, Inc., Chicago, IL, USA). Numerical data are expressed as the mean \pm standard deviation. Differences among multiple groups were analyzed with one-way analysis of variance followed by a Student's Newman Keul's-Q test.
$\mathrm{P}<0.05$ was considered to indicate a statistically significant difference.

\section{Results}

miR-124-3p was downregulated in rats with $\mathrm{CH}$. The results of RT-qPCR indicated that the expression of miR-124-3p was significantly decreased in the hippocampus tissues of rats with $\mathrm{CH}$ compared with the control group. Compared with the TH model group, the level of miR-124-3p significantly increased in the hippocampus tissues of $\mathrm{CH}$ rats treated with the miR-124-3p mimic. However, no significant differences were identified between the NC and TH groups (Fig. 1).

Bioinformatics analysis. Many potential targets of miR-124-3p were identified, however the PDCD6 3'UTR exhibited the seed sequence of miR-124-3p (Fig.2A).PDCD6 is a calcium-binding modulator protein, which regulates cell proliferation and apoptosis $(23,24)$, indicating a promising role in $\mathrm{CH}$ development. Therefore, PDCD6 was used for subsequent experiments.

miR-124-3p targeted PDCD6 3'UTR. The interaction between miRNA-124-3p and PDCD6 was verified using a dual-luciferase reporter assay system. miRNA-124-3p significantly inhibited the luciferase activity in the PDCD6-wt group but not in the PDCD6-mut group (Fig. 2B).

PDCD6 was upregulated in rats with $\mathrm{CH}$. The results indicated that the mRNA and protein levels of PDCD6 were significantly increased in the hippocampus tissues of rats with $\mathrm{CH}$ compared with that in the control group. Compared with the model group (TH), the level of PDCD6 significantly decreased in the hippocampus tissues of $\mathrm{CH}$ rats treated with the miR-124-3p mimic. However, no significant differences were identified between the $\mathrm{NC}$ and TH groups (Fig. 3).

miR-124-3p protected neurons from apoptosis. The results of flow cytometry demonstrated that thyroid hypofunction induced cell apoptosis, which was significantly inhibited by miR-124-3p mimics compared with the control group (Fig. 4). No significant difference in neuronal cell apoptosis was observed between the thyroid hypofunction and miR-124-3p NC groups (Fig. 4). The results of the flow cytometric analysis were confirmed by analyzing PARP cleavage. Cleavage of PARP was significantly increased in the thyroid hypofunction group compared with the control group and this increase was significantly reversed by transfection with miRNA-124-3p mimics. No significant difference in the expression of PARP cleavage in neuronal cells was observed between the thyroid hypofunction group and miR-124-3p NC group (Fig. 5).

miR-124-3p reversed the thyroid hypofunction-induced upregulation of caspase-3 and Bax and the downregulation of $\mathrm{Bcl}$-2. No significant difference was observed in the expression of caspase-3, Bax and Bcl-2 in neuronal cells between the thyroid hypofunction group and miR-124-3p NC group (Fig. 6). Thyroid hypofunction significantly increased the expression of caspase- 3 and Bax proteins, while the expression of Bcl-2 protein was significantly decreased compared with 

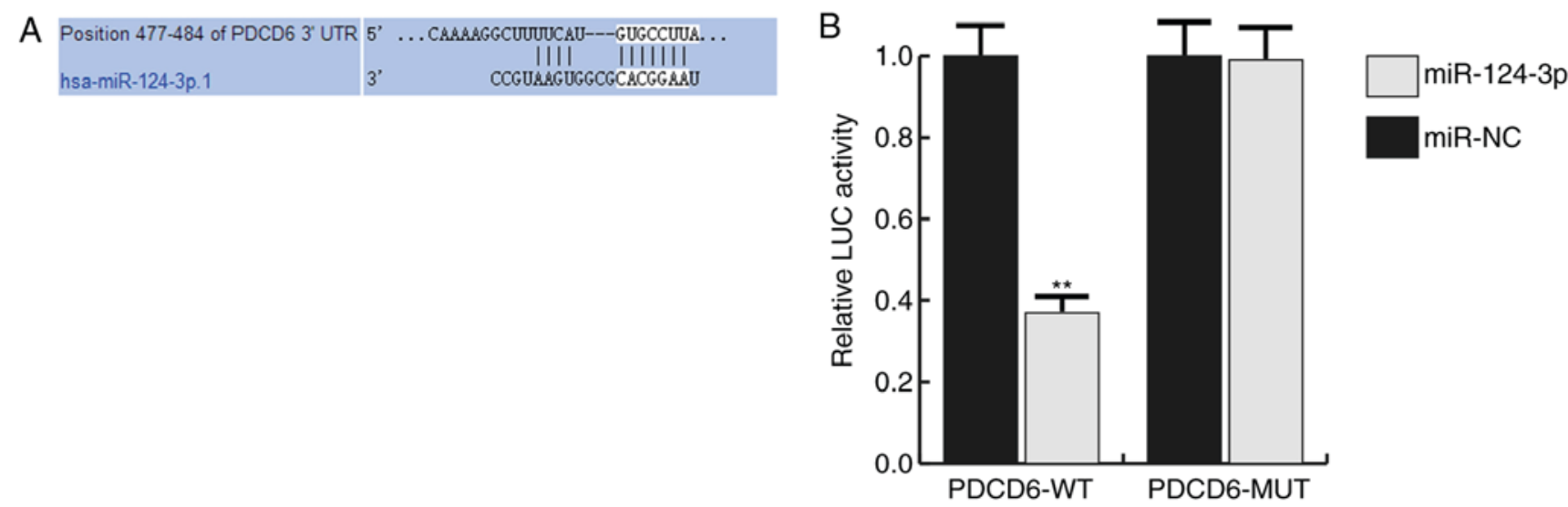

Figure 2. miR-124-3p targeted the PDCD6 3'UTR. (A) Bioinformatics analysis revealed that PDCD6 3'UTR exhibits the seed sequence of miR-124-3p. (B) miRNA-124-3p significantly inhibited the luciferase activity in the PDCD6-WT group but not in the PDCD6-MUT group. Values are expressed as the mean \pm standard deviation. Tests were performed in triplicate. ${ }^{* * *} \mathrm{P}<0.01$ vs. miR-NC. miR-124-3p, microRNA-124-3p; PDCD6, programmed cell death protein 6; UTR, untranslated region; LUC, luciferase; PDCD6-WT, PDCD6-wild type plasmid group; PDCD6-MUT, PDCD6-negative control group; miR-NC, negative control microRNA group; miR-124-3p, miR-124-3p group.

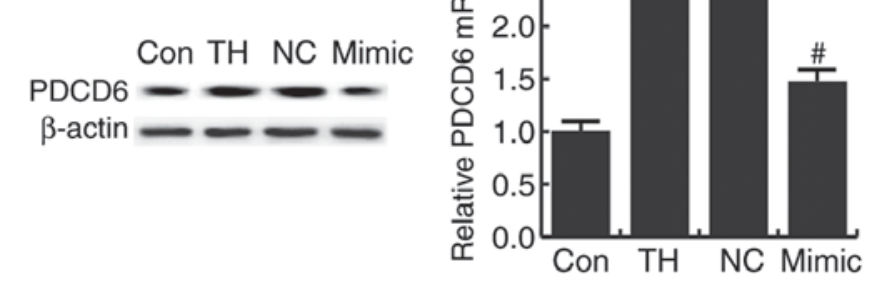

Figure 3. Western blot analysis/RT-qPCR demonstrated that PDCD6 was upregulated in $\mathrm{CH}$ rats. Values are expressed as the mean \pm standard deviation. Tests were performed in triplicate. ${ }^{* *} \mathrm{P}<0.01$ vs. the control group;

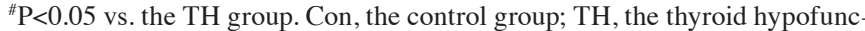
tion group; NC, the miR-124-3p NC group; mimic, the miR-124-3p mimics group; PDCD6, programmed cell death protein 6.

the control group; this effect was reversed by transfection with miRNA-124-3p mimics (Fig. 6).

\section{Discussion}

The results of the present study indicate that the expression of miR-124-3p was significantly decreased and the expression of PDCD6 was significantly increased in the hippocampus of rats with $\mathrm{CH}$ in comparison with the control group. miR-124-3p protected neurons from thyroid hypofunction-induced apoptosis. Thyroid hypofunction induced Caspase-3 and Bax protein expression and reduced $\mathrm{Bcl}-2$ protein expression. However, these changes were eliminated by miR-124-3p via the targeting of PDCD6. In conclusion, it was demonstrated that miRNA-124-3p serves a protective role in $\mathrm{CH}$.

$\mathrm{CH}$ is one of the most frequent preventable causes of mental retardation and leads to multiple developmental disorders (4). Despite early diagnosis of $\mathrm{CH}$ in infants, neurologic maldevelopment may also occur if treatment is not optimized in the first 2-3 years of life (9). Therefore, it is important for infants to receive optimized treatment.
miRNAs serve a role in nervous system diseases, including autoimmune neuroinflammation (13), Alzheimer's disease (14) and Parkinson's diseases (15). A previous study indicated that the expression of miR-124 was downregulated in newborn rats with thyroid hypofunction (19). However, the target genes of miR-124 remain unknown. Furthermore, as a sub-member of miR-124, the level and role of miR-124-3p in $\mathrm{CH}$ remains unclear. Therefore, the present study was performed to investigate the role and precise molecular mechanism of miR-124-3p in $\mathrm{CH}$.

RT-qPCR was performed to investigate changes in the expression of miR-124-3p in $\mathrm{CH}$. The results indicated that miR-124-3p was significantly decreased in the hippocampus of rats with $\mathrm{CH}$ compared with the control group, which is consistent with a previous study (19). Bioinformatics analysis was used to predict the possible target genes of miR-124-3p. The PDCD6 3'UTR exhibited the seed sequence of miR-124-3p. The interaction between miRNA-124-3p and PDCD6 was then verified using a dual Luciferase reporter assay system. PDCD6 is a calcium-binding modulator protein that regulates cell proliferation and death $(23,24)$. Aberrant expression of PDCD6 has been observed in various types of human cancer and may act as either an oncogene or a tumor suppressor. For instance, PDCD6 was recently reported to be a tumor suppressor and may be a potential therapeutic target in glioblastoma (25); reintroduction of PDCD6 significantly reverses the effects of miR-183 in enhanced cell cycle/proliferation and inhibits the apoptosis of leukemia cell lines (26); and PDCD6 was highly expressed in metastatic ovarian cancer and induced cell migration/invasion (27). In the present study, RT-qPCR was used to detect the differential expression of PDCD6 in $\mathrm{CH}$ and the results indicated that PDCD6 was significantly increased in the hippocampus of rats with $\mathrm{CH}$ compared with the control group. These results were consistent with those of a previous study (27). The results also indicated a negative correlation between miR-124-3p and PDCD6.

It has previously been demonstrated that miR-124 protects neurons against apoptosis in cerebral ischemic stroke (28). 


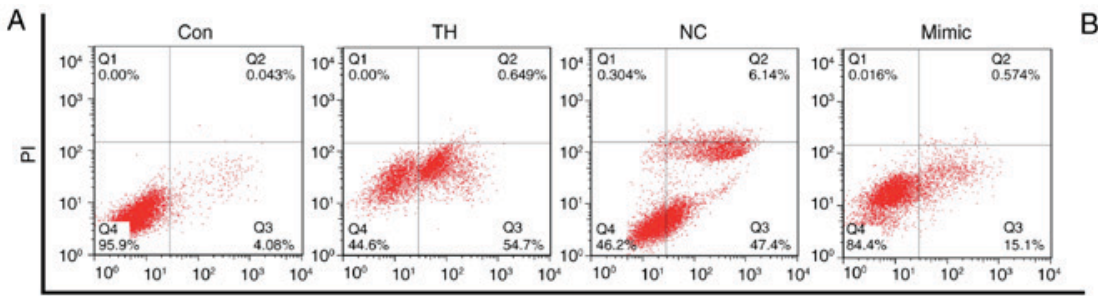

B

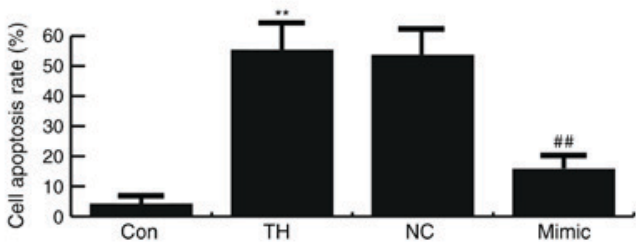

Figure 4. miR-124-3p protected neurons from apoptosis. (A) Flow cytometry analysis indicated that thyroid hypofunction induced apoptosis, while apoptosis was remarkably inhibited by miR-124-3p mimics compared with the control group. (B) Quantification of flow cytometry analysis. Values are expressed as the mean \pm standard deviation. Tests were performed in triplicate. ${ }^{* *} \mathrm{P}<0.01$ vs. the control group; ${ }^{\# \#} \mathrm{P}<0.01$ vs. the TH group. miR-124-3p, microRNA-124-3p; Con, the control group; TH, the thyroid hypofunction group; NC, the miR-124-3p negative control group; mimic, the miR-124-3p mimics group; PI, propidium iodide.
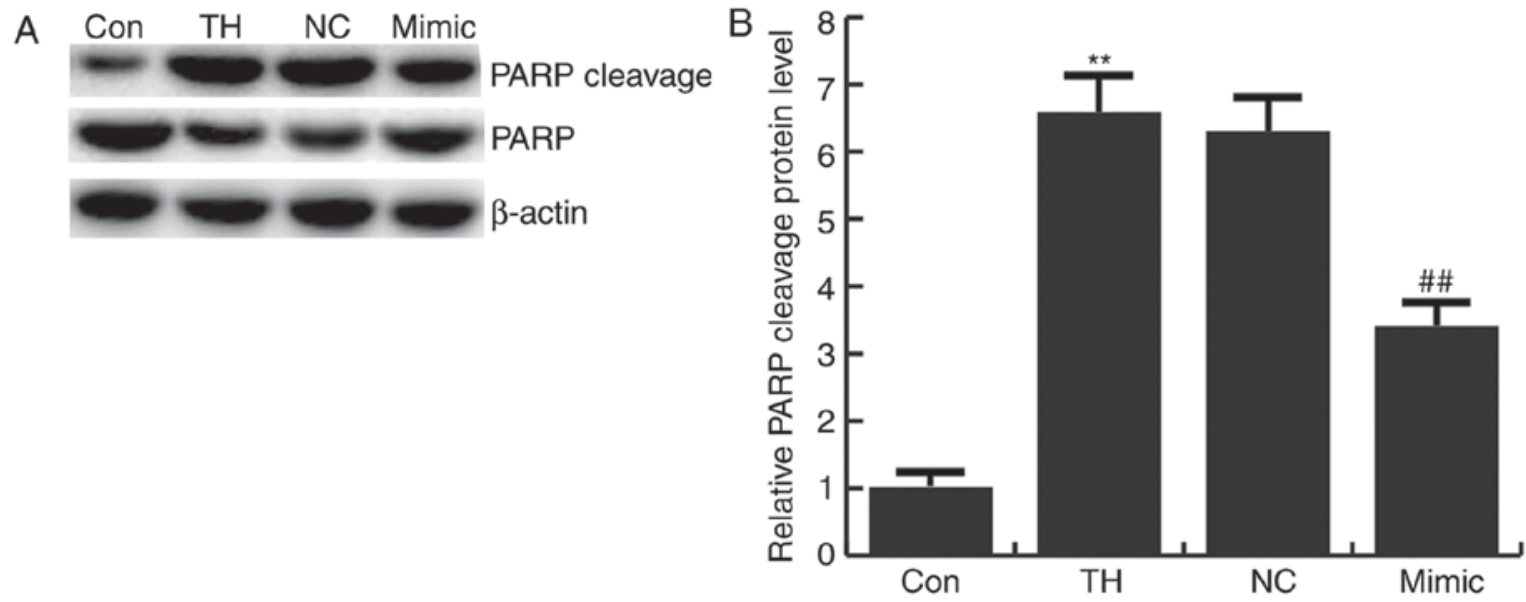

Figure 5. miR-124-3p reversed thyroid hypofunction-induced upregulation of PARP cleavage. (A) Western blot analysis of PARP expression and PARP cleavage. (B) Quantification of western blots. Values are expressed as the mean \pm standard deviation. Tests were performed in triplicate. ${ }^{* *} \mathrm{P}<0.01$ vs. the control group; ${ }^{\# \#} \mathrm{P}<0.01$ vs. the TH group. miR-124-3p, microRNA-124-3p; PARP, Poly ADP-ribose polymerase 1; con, the control group; TH, the thyroid hypofunction group; NC, the miR-124-3p NC group; mimic, the miR-124-3p mimics group.

A
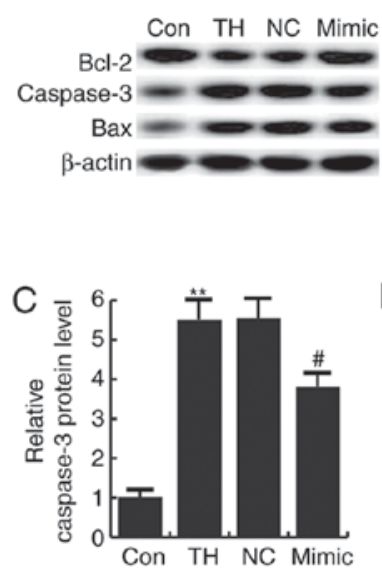

D

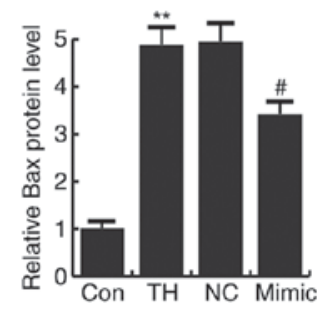

Figure 6. miR-124-3p reversed thyroid hypofunction-induced upregulation of caspase-3 and Bax, while the expression of Bcl-2 was downregulated. (A) Western blot analysis of the expression of Bcl-2, caspase-3 and Bax in each group. (B) Quantification of the expression of Bcl-2 in each group. (C) Quantification of the expression of caspase-3 in each group. (D) Quantification of the expression of Bax in each group. Values are expressed as the mean \pm standard deviation. Tests were performed in triplicate. ${ }^{* *} \mathrm{P}<0.01$ vs. the control group; ${ }^{\#} \mathrm{P}<0.05$ vs. the TH group; ${ }^{\# \#} \mathrm{P}<0.01$ vs. the TH group. miR-124-3p, microRNA-124-3p con, the control group; TH, the thyroid hypofunction group; NC, the miR-124-3p NC group; mimic, the miR-124-3p mimics group; Bcl-2, B-cell lymphoma 2; Bax, Bcl-2-associated X protein.
The results of the present study demonstrated that the thyroid hypofunction group had the highest rate of apoptosis, whereas transfection with miR-124-3p mimics significantly decreased this effect, consistent with previous studies $(19,28)$. Apoptosis-associated proteins were further detected in the present study using western blot analysis.

Caspase-3 is a member of the cysteine-aspartic acid protease family, which is encoded by the CASP3 gene (29). Caspase- 3 is activated in apoptotic cells by the extrinsic and intrinsic pathways (30). In addition, Bax was the first identified pro-apoptotic member of the Bcl-2 protein family (31) and $\mathrm{Bcl}-2$ itself is an important anti-apoptotic protein (32). In the present study, the protein levels of Caspase-3 and Bax were significantly increased, whereas the expression of Bcl-2 was significantly decreased in the thyroid hypofunction group. These effects were reversed by transfection with miRNA-124-3p mimics.

In conclusion, the results of the present study demonstrate that miRNA-124-3p serves a protective role in $\mathrm{CH}$ via targeting PDCD6. miR-124-3p may therefore be a potential therapeutic target for the treatment of infants with $\mathrm{CH}$. However, as miR-124-3p has various target genes, the role and underlying mechanism of miR-124-3p in $\mathrm{CH}$ progression remain largely unknown. Thus, further study is required to support the conclusions drawn from the present study. 


\section{References}

1. Kollati Y, Ambati RR, Reddy PN, Kumar NSS, Patel RK and Dirisala VR: Congenital hypothyroidism: Facts, Facets \& Therapy. Curr Pharm Des 23: 2308-2313, 2017.

2. Ruiz de Oña C, Obregón MJ, Escobar del Rey F and Morreale de Escobar G: Developmental changes in rat brain 5'-deiodinase and thyroid hormones during the fetal period: The effects of fetal hypothyroidism and maternal thyroid hormones. Pediatr Res 24 588-594, 1988.

3. Zhai X: Effects of thyroid hormone replacement therapy on thyroid hormone levels and electrocardiogram changes in geriatric patients with hypothyroidism. Pak J Pharm Sci (5 Suppl) 30: S1939-S1942, 2017.

4. Shukla GC, Singh J and Barik S: MicroRNAs: Processing, maturation, target recognition and regulatory functions. Mol Cell Pharmacol 3: 83-92, 2011.

5. Fisher DA: Second International conference on neonatal thyroid screening: Progress report. J Pediatr 102: 653-654, 1983.

6. Harris KB and Pass KA: Increase in congenital hypothyroidism in New York State and in the United States. Mol Genet Metab 91: 268-277, 2007.

7. Alm J, Hagenfeldt L, Larsson A and Lundberg K: Incidence of congenital hypothyroidism: Retrospective study of neonatal laboratory screening versus clinical symptoms as indicators leading to diagnosis. Br Med J (Clin Res Ed) 289: 1171-1175, 1984.

8. LaFranchi SH and Austin J: How should we be treating children with congenital hypothyroidism? J Pediatr Endocrinol Metab 20: 559-578, 2007.

9. Bongers-Schokking JJ and de Muinck Keizer-Schrama SM: Influence of timing and dose of thyroid hormone replacement on mental psychomotor, and behavioral development in children with congenital hypothyroidism. J Pediatr 147: 768-774, 2005.

10. Bartel DP: MicroRNAs: Target recognition and regulatory functions. Cell 136: 215-233, 2009.

11. Kozomara A and Griffiths-Jones S: miRBase: Integrating microRNA annotation and deep-sequencing data. Nucleic Acids Res 39 (Database Issue): D152-D157, 2011.

12. Filipowicz W, Bhattacharyya SN and Sonenberg N: Mechanisms of post-transcriptional regulation by microRNAs: Are the answers in sight? Nat Rev Genet 9: 102-114, 2008.

13. Ghorbani S, Talebi F, Chan WF, Masoumi F, Vojgani M, Power C and Noorbakhsh F: MicroRNA-181 variants regulate T cell phenotype in the context of autoimmune neuroinflammation. Front Immunol 8: 758, 2017.

14. Song J and Kim YK: Identification of the Role of miR-142-5p in Alzheimer's Disease by Comparative Bioinformatics and Cellular Analysis. Front Mol Neurosci 10: 227, 2017.

15. Grasso M, Piscopo P, Confaloni A and Denti MA: Circulating miRNAs as biomarkers for neurodegenerative disorders. Molecules 19: 6891-6910, 2014.

16. Geng L, Liu W and Chen Y: miR-124-3p attenuates MPP+-induced neuronal injury by targeting STAT3 in SH-SY5Y cells. Exp Biol Med (Maywood) 242: 1757-1764, 2017.

17. Huang S, Ge X, Yu J, Han Z, Yin Z, Li Y, Chen F, Wang H, Zhang J and Lei P: Increased miR-124-3p in microglial exosomes following traumatic brain injury inhibits neuronal inflammation and contributes to neurite outgrowth via their transfer into neurons. FASEB J: fj.201700673R, 2017.
18. Dong RF, Zhang B, Tai LW, Liu HM, Shi FK and Liu NN: The Neuroprotective role of MiR-124-3p in a 6-Hydroxydopamine-induced cell model of Parkinson's disease via the regulation of ANAX5. J Cell Biochem 116: 269-277, 2017.

19. Shao Q, Jiang W and Jin Y: MiR-124 effect in neuron apoptosis in newborn rat with thyroid hypofunction. Int J Clin Exp Pathol 8: 14465-14471, 2015.

20. Fabian ID, Rosner M, Fabian I, Vishnevskia-Dai V, Zloto O, Shinderman Maman E, Cohen K, Ellis M, Lin HY, Hercbergs A, et al: Low thyroid hormone levels improve survival in murine model for ocular melanoma. Oncotarget 6: 11038-11046, 2015.

21. Xu LJ, Ouyang YB, Xiong X, Stary CM and Giffard RG: Post-stroke treatment with miR-181 antagomir reduces injury and improves long-termbehavioral recovery in mice after focal cerebral ischemia. Exp Neurol 264: 1-7, 2015.

22. Livak KJ and Schmittgen TD: Analysis of relative gene expression data using real-time quantitative PCR and the 2(-Delta Delta C(T)) method. Methods 25: 402-408, 2001.

23. Hashemi M, Yousefi J, Hashemi SM, Amininia S, Ebrahimi M, Taheri M and Ghavami S: Association between programmed cell death 6 interacting protein insertion/deletion polymorphism and the risk of breast cancer in a sample of Iranian Population. Dis Markers 2015: 854621, 2015.

24. Zhou B, Bai P, Xue H, Zhang Z, Shi S, Zhang K, Wang Y, Wang K, Quan Y, Song Y and Zhang L: Single nucleotide polymorphisms in PDCD6 gene are associated with the development of cervical squamous cell carcinoma. Fam Cancer 14: 1-8, 2015

25. Zhang D, Wang F, Pang Y, Zhao E, Zhu S, Chen F and Cui H: ALG2 regulates glioblastoma cell proliferation, migration and tumorigenicity. Biochem Biophys Res Commun 486: 300-306, 2017.

26. Wang X, Zuo D, Yuan Y, Yang X, Hong Z and Zhang R: MicroRNA-183 promotes cell proliferation via regulating programmed cell death 6 in pediatric acute myeloid leukemia. J Cancer Res Clin Oncol 143: 169-180, 2017.

27. Su D, Xu H, Feng J, Gao Y, Gu L, Ying L, Katsaros D, Yu H, $\mathrm{Xu}$ S and Qi M: PDCD6 is an independent predictor of progression free survival in epithelial ovarian cancer. J Transl Med 10: 31, 2012.

28. Sun Y, Gui H, Li Q, Luo ZM, Zheng MJ, Duan JL and Liu X: MicroRNA-124 protects neurons against apoptosis in cerebral ischemic stroke. CNS Neuroscience Ther 19: 813-819, 2013.

29. Alnemri ES, Livingston DJ, Nicholson DW, Salvesen G, Thornberry NA, Wong WW and Yuan J: Human ICE/CED-3 protease nomenclature. Cell 87: 171, 1996.

30. Salvesen GS: Caspases: Opening the boxes and interpreting the arrows. Cell Death Differ 9: 3-5, 2002.

31. Oltvai ZN, Milliman CL and Korsmeyer SJ: Bcl-2 heterodimerizes in vivo with a conserved homolog, Bax, that accelerates programmed cell death. Cell 74: 609-619, 1993.

32. Cleary ML, Smith SD and Sklar J: Cloning and structural analysis of cDNAs for bcl-2 and a hybrid bcl-2/immunoglobulin transcript resulting from the $\mathrm{t}(14 ; 18)$ translocation. Cell 47 : 19-28, 1986. 